\title{
Transcriptome profiling reveals the response process of tomato carrying Cf-19 and Cladosporium fulvum interaction
}

Tingting Zhao', Wenhong Liu', Zhentong Zhao', Huanhuan Yang ${ }^{1}$, Yufang Bao', Dongye Zhang ${ }^{1}$, Ziyu Wang ${ }^{1}$, Jingbin Jiang ${ }^{1}$, Ying $X u^{1}$, He Zhang ${ }^{1}$, Jingfu Li ${ }^{1}$, Qingshan Chen ${ }^{1,2^{* \dagger}}$ and Xiangyang $X u^{1,3^{*}+}$ (D)

\begin{abstract}
Background: During tomato cultivation, tomato leaf mould is a common disease caused by Cladosporium fulvum (C. fulvum). By encoding Cf proteins, which can recognize corresponding AVR proteins produced by C. fulvum, Cf genes provide resistance to $C$. fulvum, and the resistance response patterns mediated by different $C f$ genes are not identical. Plants carrying the Cf-19 gene show effective resistance to C. fulvum in the field and can be used as new resistant materials in breeding. In this study, to identify key regulatory genes related to resistance and to understand the resistance response process in tomato plants carrying Cf-19, RNA sequencing (RNA-seq) was used to analyse the differences between the response of resistant plants (CGN18423, carrying the Cf-19 gene) and susceptible plants (Moneymaker (MM), carrying the (f-0 gene) at 0, 7 and 20 days after inoculation (dai).

Results: A total of 418 differentially expressed genes (DEGs) were identified specifically in the CGN18423 response process. Gene Ontology (GO) analysis revealed that GO terms including "plasma membrane (GO_Component)", "histidine decarboxylase activity (GO_Function)", and "carboxylic acid metabolic process (GO_Process)", as well as other $10 \mathrm{GO}$ terms, were significantly enriched. The "plant hormone signal transduction" pathway, which was unique to CGN18423 in the 0-7 dai comparison, was identified. Moreover, ten key regulatory points were screened from the "plant hormone signal transduction" pathway and the "plant pathogen interaction" pathway. Hormone content measurements revealed that the salicylic acid (SA) contents increased and peaked at 7 dai, after which the contents deceased and reached minimum values in both CGN18423 and MM plants at 20 dai. The jasmonic acid (JA) content increased to a very high level at 7 dai but then decreased to nearly the initial level at 20 dai in CGN18423, while it continued to increase slightly during the whole process from 0 to 20 dai in MM.
\end{abstract}

Conclusions: The initial responses are very different between the resistant and susceptible plants. The "plant hormone signal transduction" pathway is important for the formation of Cf-19-mediated immunity. In addition, both JA and SA play roles in regulating the Cf-19-dependent resistance response.

Keywords: Cf-19 gene, Solanum lycopersicum, Cladosporium fulvum, Tomato leaf mould, RNA-seq, Resistance response

\section{Background}

Tomato (Solanum lycopersicum; S. lycopersicum) leaf mould is an economically important disease that causes considerable yield losses in tomato cultivation worldwide. This disease is caused by the biotrophic fungus Cladosporium fulvum (C. fulvum) [1], which exists as many different

\footnotetext{
*Correspondence: qshchen@126.com; xxy709@126.com

${ }^{+}$Qingshan Chen and Xiangyang Xu contributed equally to this work.

${ }^{1}$ College of Horticulture and Landscape Architecture, Northeast Agricultural

University, Harbin 150030, China

Full list of author information is available at the end of the article
}

physical races and differentiates rapidly. C. fulvum resistance genes (Cf genes), which provide resistance to $C$. fulvum, were identified in wild species and subsequently bred into cultivated tomato. The use of $\mathrm{Cf}$ genes in tomato breeding represents an efficient method for controlling leaf mould disease in tomato cultivation $[2,3]$.

Plants have evolved two kinds of innate immune mechanisms: PAMP-triggered immunity (PTI) and effectortriggered immunity (ETI) $[4,5]$. PTI is a resistance reaction activated by the pattern recognition receptors (PRR) to

(C) The Author(s). 2019 Open Access This article is distributed under the terms of the Creative Commons Attribution 4.0 International License (http://creativecommons.org/licenses/by/4.0/), which permits unrestricted use, distribution, and 
identify conserved pathogen-associated molecular patterns (PAMPs). ETI was shown to depend on plant resistance proteins ( $\mathrm{R}$ proteins) to identify pathogen-secreted proteins directly or indirectly and to activate a strong resistance reaction inhibiting pathogen infection [6].

Tomato and C. fulvum are model pathosystems for the study of ETI $[1,7,8]$. The distinct recognition mechanism between Cfs and Avrs, including Cf-2/Avr2 [9] and Cf-9/Avr9 [10], has already been reported. Many studies have focused on Cf/Avr-dependent defence responses. Both Avr4/Cf-4 and Avr9/Cf-9 signalling elicit responses by the same spectrum of genes, while the Avr4/Cf-4dependent hypersensitive response (HR) is more severe than the Avr9/Cf-9-dependent HR, indicating that the distinction between the Avr4/Cf-4- and Avr9/Cf-9dependent HR most likely results from events upstream of the defence responses [11, 12]. Studies have shown that, in the Cf-12-mediated resistance response process, differentially expressed genes (DEGs) were significantly enriched in defence-signalling pathways such as the calcium-dependent protein kinase (CDPK) pathway and the jasmonic acid (JA) signalling pathway [13], and in the Cf-10-mediated resistance response process, the majority of DEGs were associated with defence-signalling pathways, including those involving oxidation-reduction processes, oxidoreductase activity and plant hormone signal transduction [14]. Additionally, many transcription factor (TF) genes were also identified in Cf-12 and Cf-10 resistance-related DEGs, indicating that TFs play an important role in the C. fulvum defence response [2].

$\mathrm{Cf}$ genes encode receptor-like proteins (RLPs), which are type I transmembrane glycoproteins that contain extracellular leucine-rich repeats (eLRRs), a transmembrane region, and a short cytoplasmic domain that exhibits no similarity to known signalling domains [1]. By recognizing Avr peptides that are secreted into the leaf apoplast during infection, $\mathrm{Cf}$ gene products provide resistance to specific races of $C$. fulvum [1]. To date, there have been at least 25 physical races of C. fulvum identified, and 10 effectors (Avr2, Avr4, Avr4E, Avr9, Ecp1, Ecp2, Ecp4, Ecp5, Ecp6 and Ecp7) have been cloned [15-23]. Twenty four $\mathrm{Cf}$ genes have been mapped onto 12 chromosomes, and 6 Cf genes (Cf-2 (Hcr2-2B, Hcr2-2C), Cf-5, Cf- 4 (Hcr9-4D), Cf-4E (Hcr9-9B), Cf-9 (Hcr9-9C), 9 DC (Hcr9M205) [18-22, 24] and 4 Cf-Ecp genes (Cf-Ecp1, Cf-Ecp2, Cf-Ecp3 and Cf-Ecp5) have been cloned [2, 25, 26].

The Cf-19 gene is a very efficient resistance gene, with no infection reports to date. This gene has been mapped to the short arm of chromosome 1, and a candidate gene, Solyc01g006550.2.1 (an allele of Cf-0), was screened within the Cf-4/9 locus as a novel homologue of the Cladosporium resistance gene Cf-9 (Hcr9) [27]. Our previous study also showed that $\mathrm{Cf}-19$ induced a marked HR in tomato plants inoculated with $C$. fulvum physiological race
1.2.4.5 [28]. In that study, we utilized the cDNA-amplified fragment length polymorphism (cDNA-AFLP) technique combined with bulk segregant analysis (BSA) to isolate tomato genes related to the $\mathrm{Cf}$-19-mediated resistance response. Twenty-six differentially expressed transcriptderived fragments (TDFs) that were upregulated in the Cf19-mediated HR process were isolated. The expression patterns of most of the genes detected by the transcript profiling of the Cf-19-mediated defence response were similar between the Cf-4- and Cf-9-mediated defence responses. SAMDC (TDF35) and Eli3 (TDF49) were first detected in the $\mathrm{Cf} / A v r$ interaction [28]. Because of the limitation of cDNA-AFLP technology, we did not obtain enough information from the study. With the development of second-generation sequencing technology, RNA sequencing (RNA-seq) has become an efficient way for the analysis of host-pathogen interactions. The successful application of RNA-seq in plant host-pathogen interaction analyses, especially in tomato (carrying Cf- 12 or Cf10)-C. fulvum $[13,14]$ and tomato-Stemphylium lycopersici [29] interaction analyses, allows a feasible way to study the Cf-19-mediated resistance response process. In the present study, RNA-seq technology will be used to analyse the Cf-19-mediated resistance response process. The results will help us further understand tomato defence mechanisms and will provide a basis for discovering new ways of controlling tomato leaf mould disease.

\section{Results}

\section{Artificial inoculation}

As shown in Fig. 1, at 7 days after inoculation (dai), no notable signs were observed on the leaves of CGN18423 (Cf-19), while the leaves of the susceptible line Moneymaker (MM) became chlorotic and slightly wilted. At 20 dai, some significant chlorotic spots, which are considered HR signs, were observed on the leaves of CGN18423 plants, and abundant mould grew on both sides of MM leaves. These classic symptoms indicated that our artificial inoculation was successful and that the plant materials collected at different time points could be used for subsequent analyses.

\section{Illumina sequencing, read mapping and identified transcripts}

In this project, 18 samples were sequenced using an Illumina HiSeq platform, with an average of $4.40 \mathrm{~Gb}$ generated. More than $97 \%$ of reads were $Q>20$, and $92 \%$ of these clean reads were $>$ Q30 (Additional file 1: Table S1). The data with a quality score $>$ Q30 were used for subsequent analyses. After filtering, an average of 29.32 million clean reads was generated, and at least $85 \%$ of these reads were mapped to the tomato reference genome (ftp://ftp.solgenomics.net/tomato_genome/assembly/build_3.00/); of these, more than $83 \%$ of the clean 

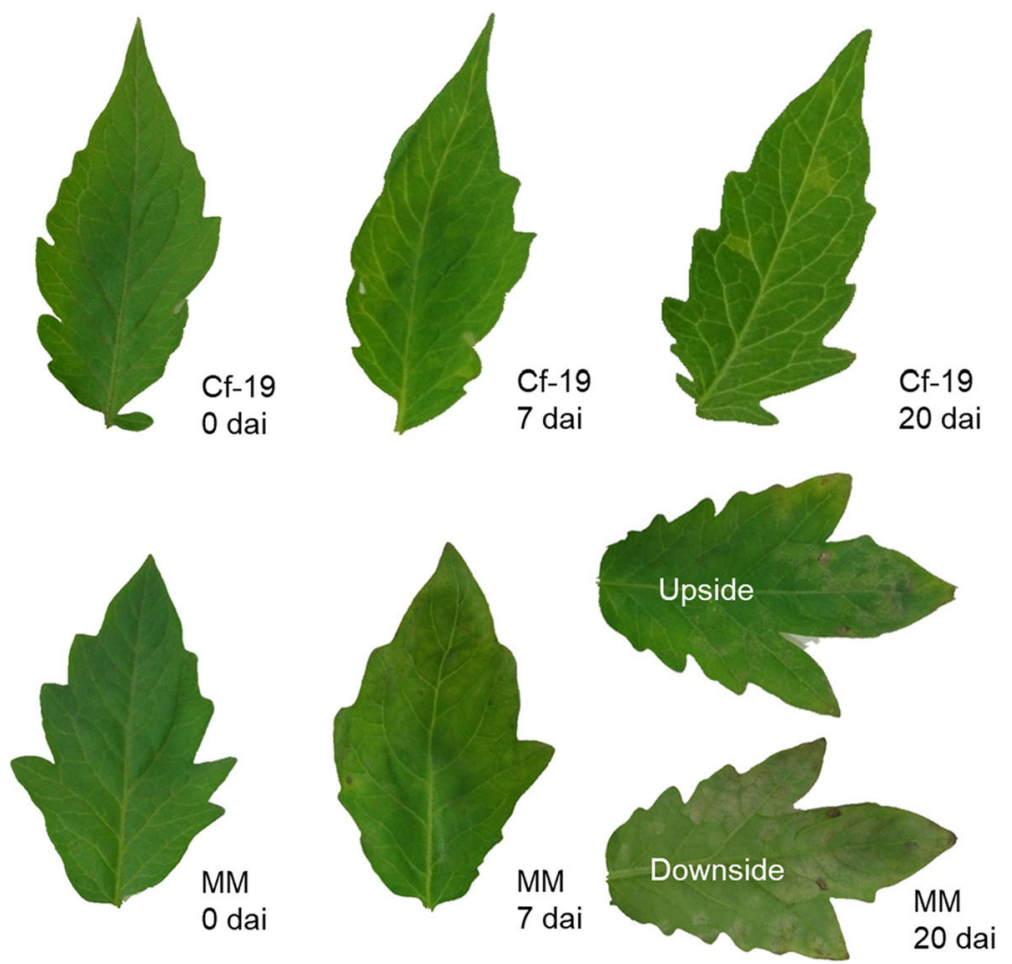

Fig. 1 C. fulvum infection symptoms on the leaves of CGN18423 and MM plants. No notable signs were observed on Cf-19 leaves at 7 dai, while significant chlorotic spots were observed on Cf-19 leaves at 20 dai. Slight chlorosis and shrinkage were observed for MM leaves at 7 dai; abundant mould grew on both sides of MM leaves at 20 dai. Cf-19: CGN18423 plants; MM: Moneymaker plants. dai: days after inoculation

reads were uniquely mapped reads. After transcript reconstruction, a total of 35,136 novel transcripts, including 17,690 unknown splicing events for known genes, 1123 novel coding transcripts without any known features, and 16,323 long noncoding RNAs were obtained.

\section{DEGs in response to $C$. fulvum inoculation}

DEGs between samples (Fig. 2) were detected based on the gene expression results. During the first stage from 0 to 7 dai, more upregulated DEGs than downregulated DEGs were detected in both MM and CGN18423 plants. During the second stage from 7 to 20 dai, fewer upregulated DEGs than downregulated DEGs were detected in both MM and CGN18423 plants. During the whole plantpathogen interaction process, downregulated DEGs were much more prevalent than upregulated DEGs in MM, while upregulated DEGs were much more prevalent than downregulated DEGs in CGN18423 plants.

\section{Verification of DEG expression patterns}

To verify the accuracy of the DEG expression patterns in RNA-seq data, quantitative real-time PCR (qRT-PCR) was used to analyse the expression patterns of 15 genes that were selected randomly from the DEGs. The plant samples used for RNA-seq, which included leaves collected from CGN18423 and MM plants at 0, 7 and 20 dai, were used for this analysis. Expression patterns for both CGN18423 (0, 7 and 20 dai) and MM (0, 7 and 20 dai) were analysed for each gene. Correlation coefficients between the RNA-seq and qRT-PCR results were calculated, and the results showed that the pairwise correlation coefficients ( $\mathrm{R}^{2}$ values) were between 0.94 and 1.0 (Fig. 3). These results reflect the high quality of our RNA-seq data, which could be used in subsequent analyses.

Gene ontology (GO) enrichment and Kyoto encyclopedia of genes and genomes (KEGG) pathway analyses

The GO classification results are shown in Additional file 3: Figure S1. DEGs at different stages of different plants were enriched in three ontology categories: biological process, cellular component and molecular function. Compared with CGN18423, MM presented more genes associated with almost all GO terms, except for the GO term "cell killing" in the 7-20 dai comparison. The number of genes associated with most GO terms, which increased in the second stage of plant-pathogen interactions in MM plants, decreased in CGN18423 plants. A total of 418 DEGs were identified specifically in the CGN18423 response process. GO analysis revealed that GO terms including "kinesin complex (GO_Component)", "microtubule motor activity (GO_Function)", and "microtubule-based movement (GO_ 


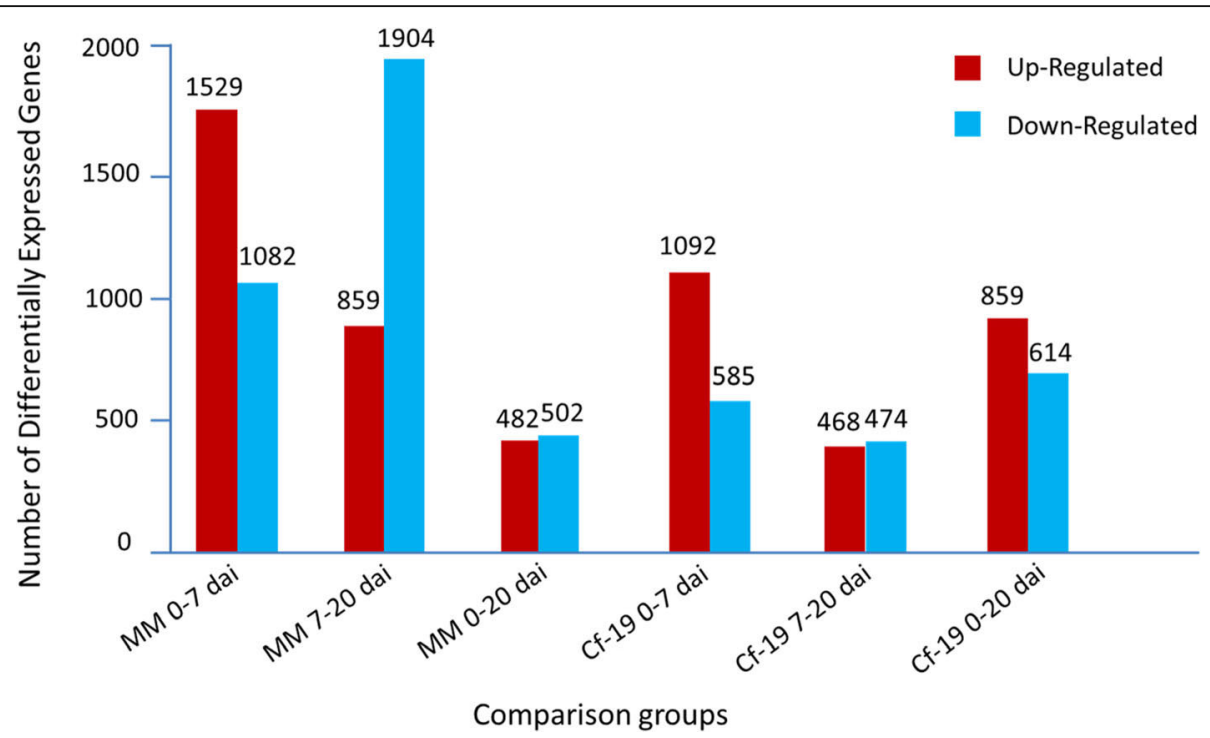

Fig. 2 Statistics of DEGs that exhibit different expression patterns. 0-7 dai: comparison between 0 and 7 dai; 7-20 dai: comparison between 7 and 20 dai; $0-20$ dai: comparison between 0 and 20 dai; dai: days after inoculation

Process)", as well as 10 others ( $\mathrm{Q}$ value $<0.05)$, were significantly enriched (Fig. 4).

Pathways with $P$-values $<0.05$ were screened and used for comparative analyses. As shown in Fig. 5, more pathways were detected in the $0-7$ dai comparison than in the 0-20 dai comparison in both MM and CGN18423 plants. Twenty pathways were common between the MM and CGN18423 plants in the $0-7$ dai comparison, and 4

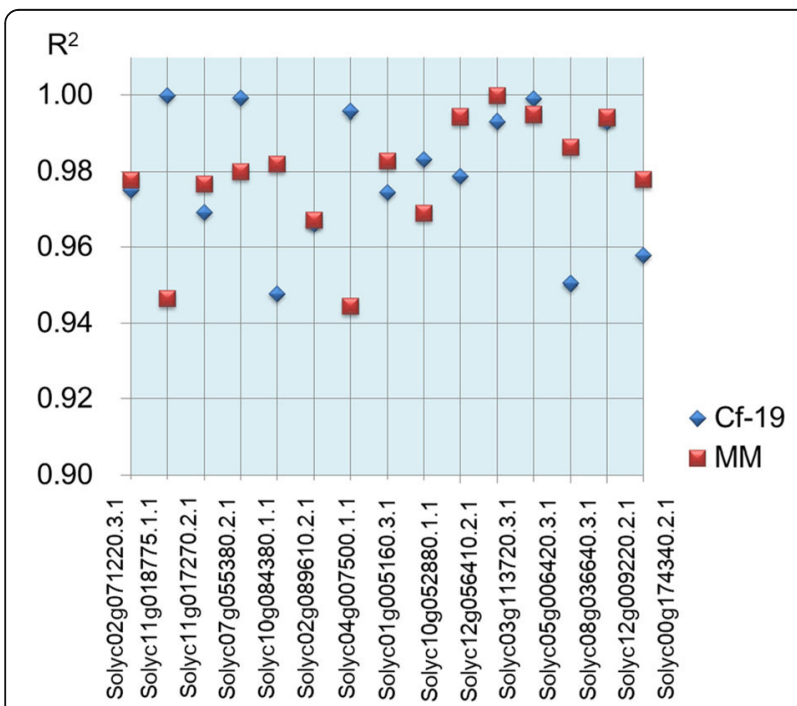

Fig. 3 Correlation coefficients between the RNA-seq and qRT-PCR results. The expression patterns for both $\mathrm{Cf}-19(0,7$ and 20 dai) and MM $(0,7$ and 20 dai) were analysed for each gene. The results obtained from both methods (RNA-seq and qRT-PCR analysis) were used to calculate correlation coefficients ( $R^{2}$ values). Each point in

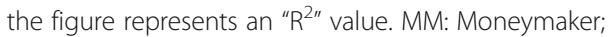
Cf-19: CGN18423 common pathways were detected between $M M$ and CGN18423 plants in the 0-20 dai comparison. Seven and two pathways were unique to $\mathrm{MM}$ in the $0-7$ dai and $0-20$ dai comparisons, respectively. The samples of CGN18423 at both of those stages had only one unique pathway. The "plant hormone signal transduction" pathway, which plays an important role in plant resistance to pathogens, is unique to CGN18423 in the 0-7 dai comparison.

\section{Identification of key regulatory points from important pathways}

"Plant hormone signal transduction" was the only pathway that was unique to CGN18423 in the 0-7 dai comparison; moreover, because "plant pathogen interaction" is an important pathway in all plant-pathogen interaction studies, we focused on these two pathways in this section. Pathway regulatory points containing genes that exhibit upregulated expression patterns in CGN18423 in the 0-7 and 0-20 dai comparisons (vertical comparisons) or that exhibit upregulated expression patterns in CGN18423 plants compared to MM plants at the same interaction stages (lateral comparisons) were identified as important regulatory points. The details of the comparison process and information are indicated on the pathway maps from the KEGG analysis (map04075, plant hormone signal transduction; map04626, plant-pathogen interaction). As shown in Additional file 4: Figure S2, "SAUR" in the "tryptophan metabolism" pathway, "A-ARR" in the "zeatin biosynthesis" pathway, "BRI1 " in the "brassinosteroid biosynthesis" pathway and "JAZ" in the " $\alpha$-linolenic acid metabolism" pathway were identified from the "plant hormone signal transduction" pathway based on the above-mentioned resistance-related expression patterns. In the "plant pathogen interaction" pathway 


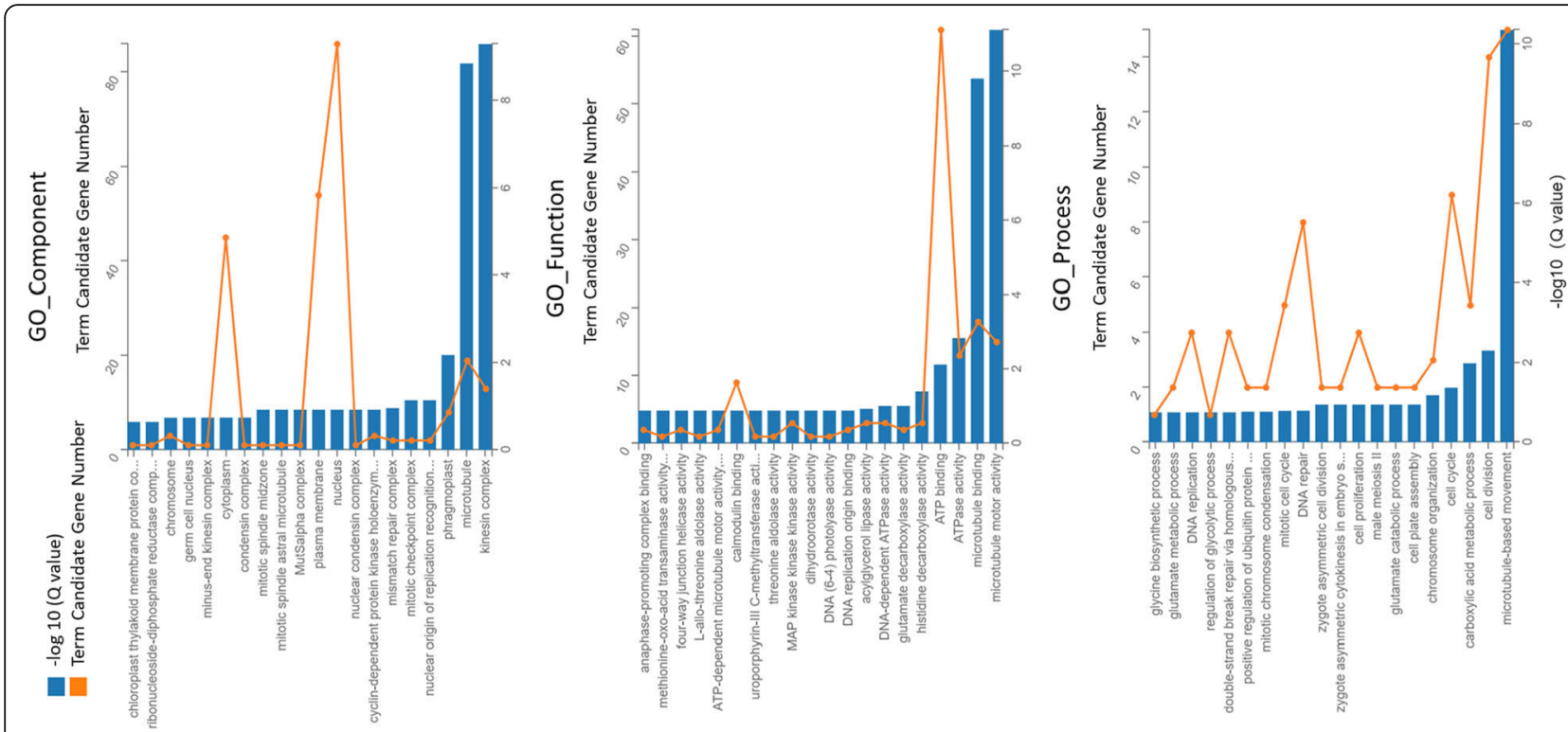

Fig. 4 GO enrichment of 418 differentially expressed genes (DEGs) identified specifically in the CGN18423 response process

(Additional file 5: Figure S3), "CaMCML”, "WRKY25/22", "WRKY28/33", "CERK1", "RRS1-R" and "WRKY1/2" were identified.

\section{Analysis of gene expression patterns at key regulatory points}

Further analysis of the gene expression patterns at the key regulatory points was carried out. The detailed expression information is shown in Fig. 6 as heat maps. Genes at different regulatory points exhibited different expression patterns among 6 comparisons. Most genes exhibited significantly upregulated expression patterns in the "R 0-7", "R 0-20", "S-R 0-7" and "S-R 0-20" comparisons, especially in the " $\mathrm{R} 0-7$ " comparison. Of all the regulatory points in the "plant hormone signal transduction pathway", genes associated with "A-ARR" and "JAZ" exhibited more uniform gene expression pattern cluster results, which means that the response patterns of the genes at these two points were similar (upregulated or downregulated) and that the genes may have similar functions. Of all the regulatory points in the "plant pathogen interaction pathway", "CaM/CML" is the largest point, containing 23 DEGs. The expression patterns of most genes at this point were upregulated in the 0-7 dai comparison in both the susceptible and resistant plants and in the 0-20 dai comparison in the resistant plants. Three WRKY-related regulatory points, which contain 24 genes in total, were identified in the "plant pathogen interaction pathway". Most WRKY genes exhibited significantly upregulated expression patterns in the 0-7 dai comparisons, while the upregulation of the same genes was more significant in the resistant plants than in the susceptible plants.

\section{Hormone measurements}

The results of "key regulatory points" identification in the "plant hormone signal transduction pathway" and gene expression pattern analyses indicate that "JAZ" is an important factor involved in the resistance response process. In addition, changes in "JAZ" may affect JA and salicylic acid (SA) functions and contents, and JA and SA are very important hormones involved in plant immunity. To further explore the relevant phenomenon behind changes in "JAZ", we measured SA and JA contents. The results showed that the SA and JA contents fluctuated differently in the different plants in response to inoculation (Fig. 7). The SA contents increased and peaked at 7 dai, after which the contents deceased and reached minimum values in both CGN18423 and MM plants at 20 dai. Although the change patterns are similar between the resistant and susceptible plants, there was more SA in the resistant plants than in the susceptible plants. The JA content differed dramatically between the resistant and susceptible plants; the JA content increased to a very high level at 7 dai but then decreased to nearly the initial level at 20 dai in CGN18423, while it continued to increase slightly during the whole process from 0 to 20 dai in MM.

\section{Discussion}

Gene expression changed most actively at the early stage of plant-pathogen interaction

Throughout the whole process of plant-pathogen interactions, the seventh day after C. fulvum inoculation is an important time point; this point separates the whole interaction process into two stages, the early stage and the late stage, according to the appearance of necrotic lesions, which were qualified as hypersensitive-like symptoms, and 


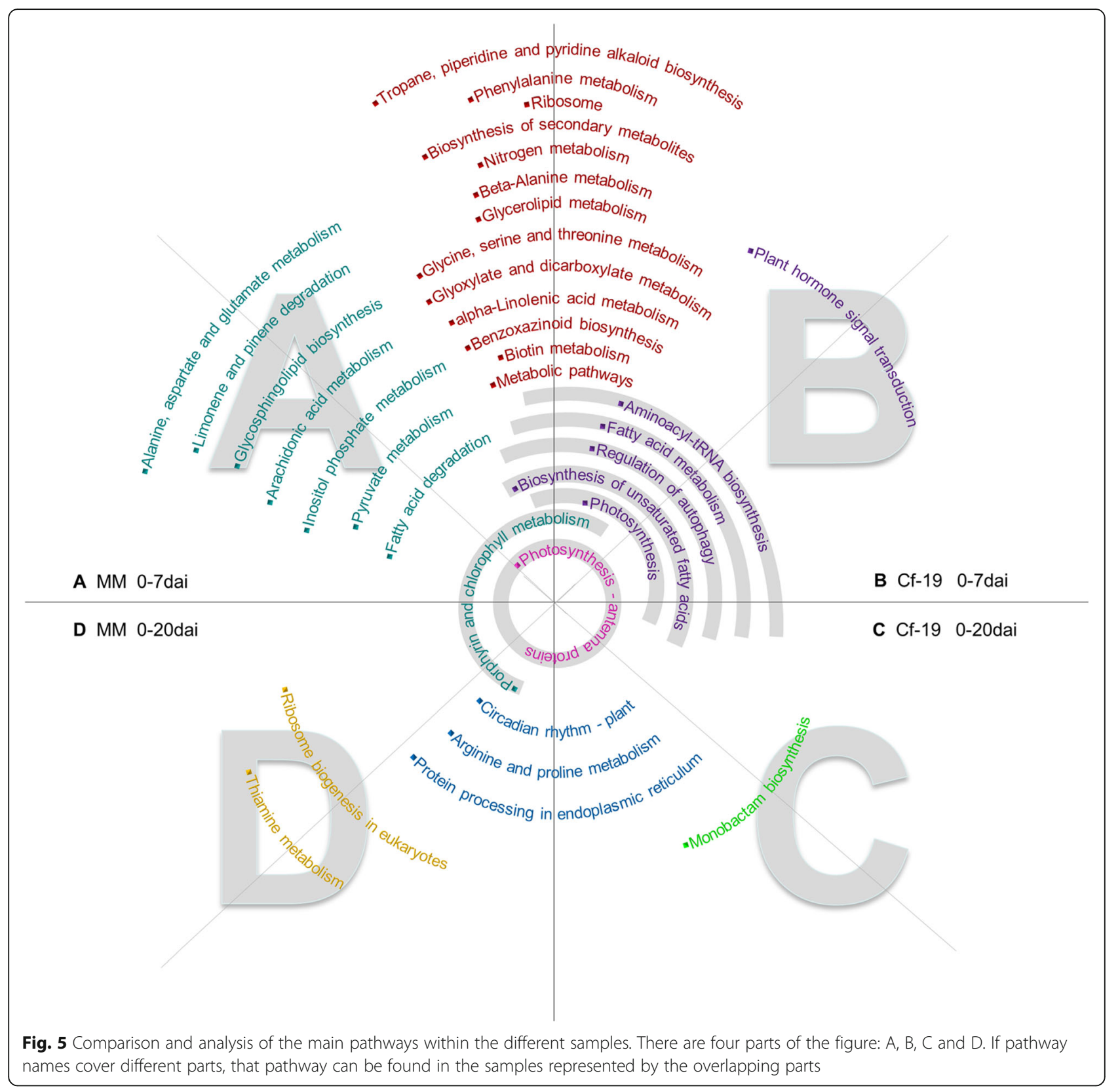

according to the results of gene expression changes [28]. On the basis of the different stages and different materials, two kinds of comparisons, vertical comparisons (gene expression comparisons between resistant plants and susceptible plants at the same time points) and horizontal comparisons (gene expression comparisons within the same plants between different time points), were carried out. The results showed that, in the vertical comparisons, there were more DEGs in the susceptible plants than in the resistant plants at the early stage, while fewer DEGs were detected in the susceptible plants than in the resistant plants at the late stage. Gene expression changes were relatively small in the resistant plants compared to the susceptible plants. In the horizontal comparisons, the gene expression patterns were very different between the early stage and late stage in both resistant and susceptible plants. In general, the expression levels of many genes changed at the early stage, although the expression levels of some of these genes recovered to their initial levels at the late stage. These comparison results indicated that, compared with the susceptible plants, the performance of the resistant plants was relatively stable and that the early stage represents very important response processes for both compatible interactions and incompatible interactions. Similar results were also reported for other $\mathrm{Cf}$ gene-mediated resistance responses [13, 14] and other plant-pathogen interactions $[29,30]$. 


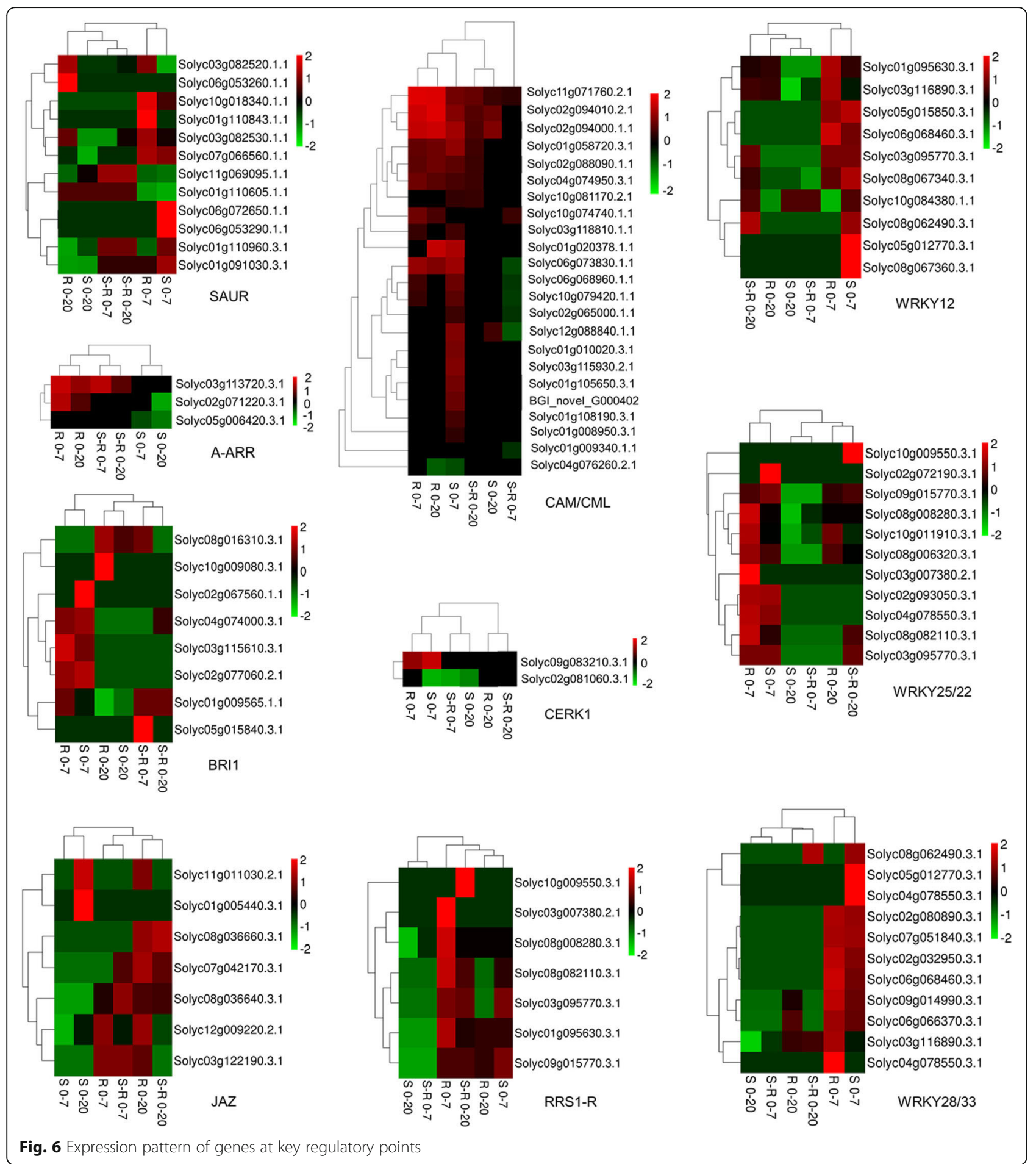

Cf-19-mediated resistance was regulated by both SA and JA We measured SA and JA contents at 0, 7 and 20 dai and found that the JA content increased to very high levels at 7 dai in the resistant plants. This interesting phenomenon was also reported for the Cf-10-mediated resistance response [14]. Furthermore, the expression levels of genes at the "JAZ" protein point were upregulated significantly in the resistant plants at 7 and 20 dai. A "JAZ" gene, Solyc12 g009220.1, whose expression was upregulated at 7 and 20 dai in this study, was also found to be upregulated in Cf-12 tomato plants after C. fulvum infection [13]. "JAZ" is one of the four key regulatory points we screened in this study. JAZs are direct targets of the $\mathrm{SCF}^{\mathrm{COI} 1} \mathrm{E} 3$ ubiquitin ligase and negatively regulate the key transcriptional activator of 


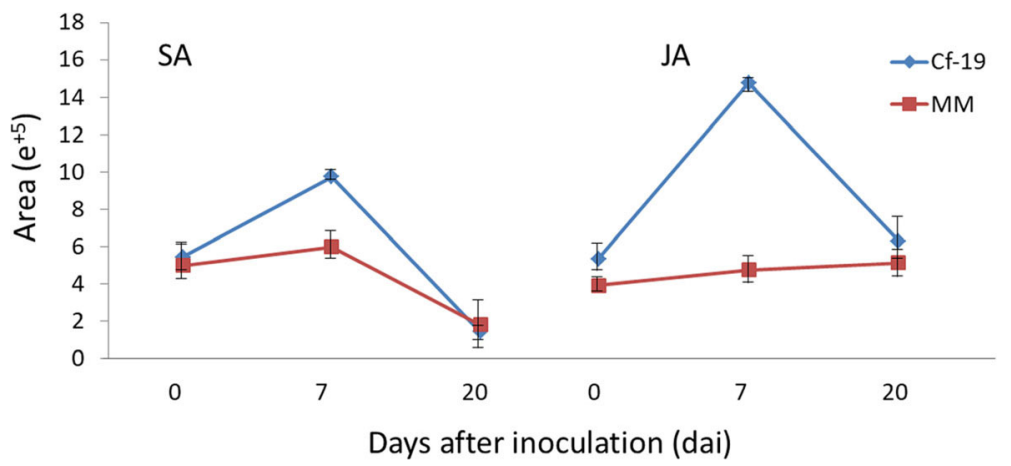

Fig. 7 Fluctuations of SA and JA after C. fulvum infection in CGN18423 plants and MM plants. SA: salicylic acid, JA: jasmonic acid, Cf-19: CGN18423, MM: Moneymaker

jasmonate responses, MYC2 [31]. JAZ protein expression suppresses JA activation and indirectly promotes SA activation. Our results indicated that both the JA and SA hormone signalling pathways are involved in the Cf-19mediated resistance response and that "JAZ" plays a very important role in regulating JA and SA during the whole defence process.

\section{Other important hormone regulation involvement in Cf- 19-mediated resistance}

Plants produce a wide variety of hormones, including auxins (Auxins), gibberellins (GAs), abscisic acid (ABA), cytokinins (CKs), SA, ethylene (ET), JA, brassinosteroids (BRs) and peptide hormones. The infection of plants by various pathogens results in changes in the levels of various phytohormones $[32,33]$. In the present study, the "plant hormone signal transduction" pathway, identified from pathways with a $P$-value $<0.05$, was unique to CGN18423 in the 0-7 dai comparison. DEGs were detected in pathways of eight different hormones, including Auxin, CK, GA, ABA, ET, BR, JA and SA, which revealed a complex signal transduction network. Comparison analysis revealed four key regulatory points for the Auxin, CK, BR and JA hormone signalling pathways based on their resistance response-related change patterns. In addition to the abovementioned "JAZ", three other key regulatory points in the "plant hormone signal transduction" pathway were identified: "SAUR" in the "tryptophan metabolism" pathway, "A$A R R$ " in the "zeatin biosynthesis" pathway and "BR-INSENSITIVE 1 (BRI-1)" in the "brassinosteroid biosynthesis" pathway. All these key points were involved in plant cell morphological changes, such as cell enlargement and cell division. These results suggest that cell morphological changes are also related to the defence process.

\section{Early $\mathrm{Ca}^{2+}$ signalling processes are different between} compatible interactions and incompatible interactions Calmodulin (CaM) and calmodulin-like (CML) proteins are two kinds of calcium $\left(\mathrm{Ca}^{2+}\right)$-sensing proteins that are involved in $\mathrm{Ca}^{2+}$ signalling processes. These proteins play important roles in plant immunity, especially during the early stage of the resistant response [34]. In this study, the most marked difference in the expression patterns of CaM/CML genes appeared in the 0-7 dai comparison between resistant plants and susceptible plants. The expression levels of many genes were upregulated significantly in the susceptible plants but not in the resistant plants, suggesting that calcium signal transduction during the initiation of pathogen infection differs between CGN18423 and MM plants. Previous studies reported PAMP-induced calcium spiking differs from that induced during symbiosis $[35,36]$. Our result may indicate that effector-induced calcium spiking also differs from that induced during symbiosis.

\section{PTI is involved in the resistance response of tomato carrying Cf-19 and C. fulvum interaction}

There are two levels of defence responses triggered by microbial recognition in plants. The first level is PAMP, which leads to PTI [37]. The second level involves intracellular immune receptors that recognize pathogen virulence effectors secreted within host cells, thereby inducing ETI [4]. The Cf/Avr interaction is a model for ETI studies, while the expression levels of many BRI1 DEGs and CERK1 DEGs, which are important components of PTI [38-40], were upregulated significantly in the $0-7$ dai comparisons in the present study. The expression patterns of these DEGs are consistent with those during the PTI process [39, 41]. Members of the complex family of WRKY TFs have been implicated in the regulation of transcriptional reprogramming associated with plant immune responses. In this study, the expression of many WRKY TFs was found to be upregulated very significantly during the early stage of interaction. Some of these WRKY TFs play important roles in the PTI process [42-45]. The mentioning of all of the PTI-related genes above indicates that PTI is involved in the early response in plants that carry Cf- 19 and that are infected with C. fulvum. 


\section{A presumed model of the Cf-19-dependent resistance response}

On the basis of the findings in the present study and in the literature on plant immunity $[46,47]$, we speculated about the general process of the Cf-19-dependent resistance response. As shown in Fig. 8, when plants carrying Cf- 19 were infected by the C. fulvum pathogen, both ETI and PTI were triggered. ETI was triggered by the recognition between AVR and the Cf-19 protein, and PTI was triggered by the recognition between CERK and chitin. The recognition was followed by an influx of $\mathrm{Ca}^{2+}$, some of which bound to different CAM/CML proteins to active downstream signalling pathways. The increase in cytosolic $\mathrm{Ca}^{2+}$ concentration could also create the proper conditions for the activation of CDPK signalling components. Mitogen-activated protein kinase (MAPK) modules could also be activated following upstream changes, including activation of heat shock protein 90 (HSP90). Many TFs, especially WRKYs, accumulated and indirectly regulated the expression of defence-related genes. These changes ultimately resulted in the expression of defence-related genes and the HR. During the whole resistance response process, both SA and JA play important regulatory roles, and JAZ is a key factor in maintaining the balance between these two hormones. Additionally, CKs, BRs, Auxin and ET play important roles in the resistance response by altering cell morphology or by affecting other cross-talk involved in the hormone regulatory network.

\section{Conclusions}

In this study, a total of 35,136 novel transcripts were obtained after transcriptome reconstruction. DEG analysis revealed that the initial responses are very different between the resistant and susceptible plants and are very important for plant immunity. Moreover, early $\mathrm{Ca}^{2+}$ signalling processes are different between compatible interactions and incompatible interactions. The "plant hormone signal transduction" pathway and the "plant pathogen interaction" pathway, which contain ten key regulatory points, are important for the Cf-19-mediated

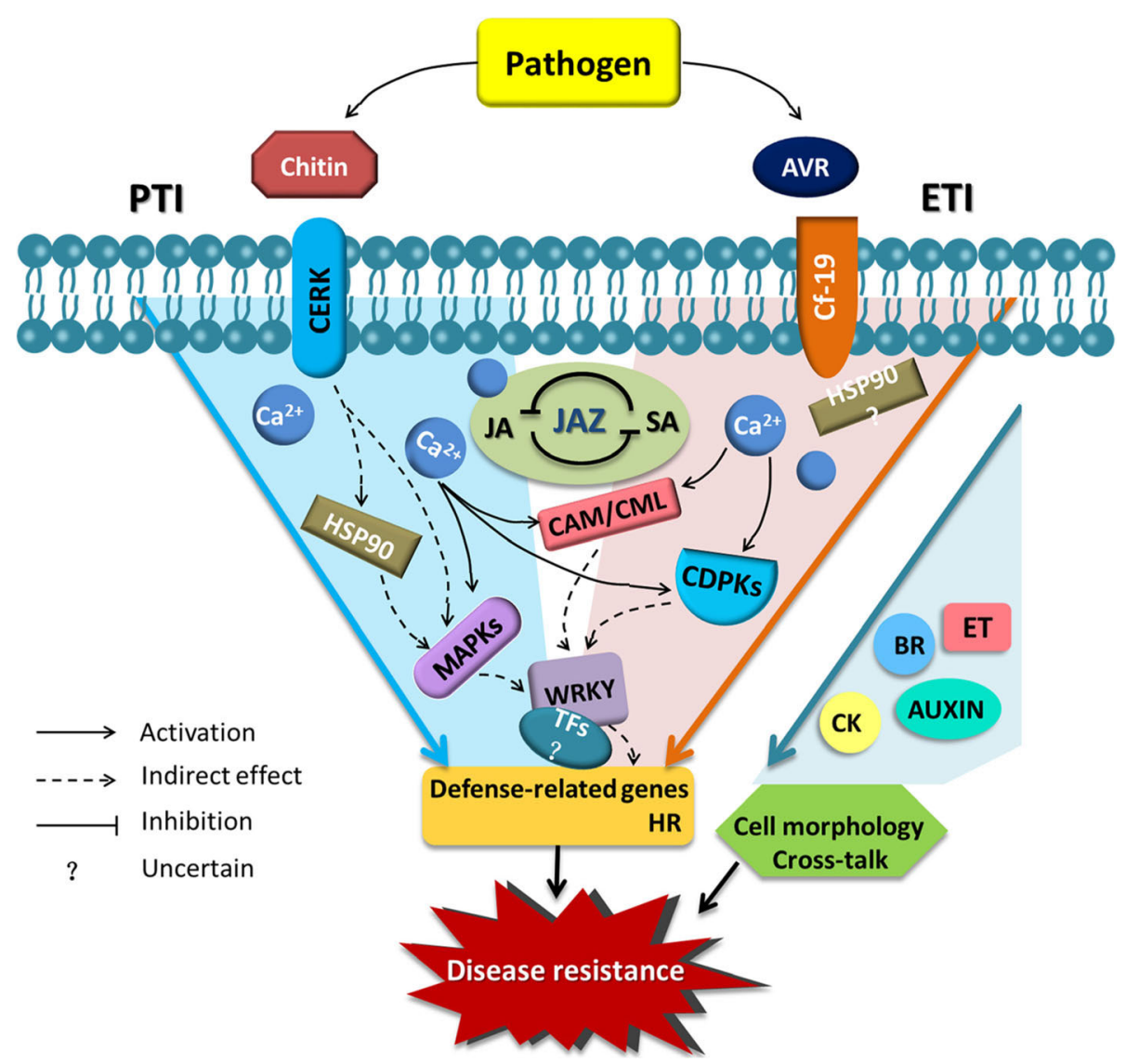

Fig. 8 Model of the resistance response of tomato plants that carry Cf-19 and that are infected with C. fulvum. Both PTI and ETI are involved in the resistance response. The balance between JA and SA, which is maintained by JAZ, is a main regulatory factor in the response process. PAMP: pathogen-associated molecular pattern; PTI: PAMP-triggered immunity; ETI: effector-triggered immunity; CAM: calmodulin; CML: calmodulin-like; MAPK: mitogen-activated protein kinases; CDPK: calcium-dependent protein kinase; HSP90: Heat shock protein 90; JA: jasmonic acid; SA: salicylic acid; JAZ: jasmonate ZIM-domain; GA: gibberellin; ABA: abscisic acid; CK: cytokinin; ET: ethylene; BR: brassinosteroid; HR: hypersensitive response 
resistance response, especially at the initial stage, and both PTI and ETI are involved in the resistance response. Last, the balance between JA and SA maintained by JAZ proteins may play a key role in regulating the Cf19-dependent resistance response.

\section{Methods}

\section{Plant material treatments}

The resistant line CGN18423 (carrying the Cf-19 gene, provided by the Institute of Vegetable and Flowers, Chinese Academy of Agricultural Sciences) and the susceptible line MM (carrying the Cf- 0 gene) were grown at Horticultural Experimental Station of Northeast Agricultural University. Thirty CGN18423 seedlings and 30 MM seedlings at the five-to-six-leaf stage were inoculated with $C$. fulvum race 1.2.3.4. Leaf samples were collected before inoculation and at 7 and 20 dai. All the leaf samples were immediately frozen in liquid nitrogen and stored at $-80^{\circ} \mathrm{C}$ prior to RNA extraction. Three biological replicates per treatment were prepared.

\section{RNA extraction and Illumina sequencing}

Total RNA was extracted from the leaf samples using a plant RNA mini kit (Watson, China) according to the manufacturer's instructions. The extracted total RNA was given to BGI Tech (Shenzhen, China) company for highthroughput sequencing. The following detailed experimental methods were provided by the company. The mRNA samples were isolated from the total RNA samples (treated with DNase I) by using oligo (dT). Eighteen cDNA libraries were constructed using purified mRNAs. The libraries were then sequenced using an Illumina HiSeq4000 machine according to the Illumina protocols [13, 48].

\section{Mapping of RNA-seq reads and DEG identification}

The reads were filtered by SOAPnuke software. Reads with adapters or with a sequencing quality less than 5 were removed $[13,48]$. The filtered reads were mapped to the $S$. lycopersicum genome (Tomato Genome Consortium, 2012). Gene expression levels were quantified by normalizing read counts to the aligned fragments per kilobase of transcript per million mapped reads (FPKM) $[49,50]$.

Hierarchical indexing for spliced alignment of transcripts (HISAT) was used to align paired-end clean reads to the reference genome $[13,51]$. The NOIseq methods in conjunction with a noisy distribution model were used to detect DEGs $[52,53]$. The false discovery rate (FDR) with a corrected $P$ value $\leq 0.05$ and an absolute value of $\log 2$ Ratio $\geq 1$ were used as the thresholds to define significant DEGs [54]. Novel transcripts were reconstructed using StringTie [55].

\section{GO enrichment and KEGG analyses of DEGs}

Significantly enriched GO terms $(P$-value $<0.05)$ were identified by the GOseq R package analyses [56]. Significantly enriched KEGG metabolic pathways were detected by using a hypergeometric test of KOBAS (v2.0) (http://kobas.cbi.pku.edu.cn/) analyses [57].

\section{qRT-PCR analysis}

Fifteen DEGs were selected for expression verification. The primers (Additional file 2: Table S2) were designed with Primer 5.0 software. qRT-PCR (including the reaction mixture and thermal conditions) was performed in accordance with the methods of our previous study [27]. The reactions were carried out on an iQ5 system (BioRad, USA). The data were analysed via the $2^{-\Delta \Delta C T}$ method [58], with $E F \alpha 1$ gene used for normalization [59].

\section{Hormone measurements}

Endogenous SA and JA were extracted from leaves using the modified methods of Llugany et al. (2013) [60] and Liu et al. (2018) [14]. The SA and JA concentrations were subsequently measured using liquid chromatographymass spectrometry (LC-MS) according to the manufacturer's instructions (AB Sciex QTRAP 5500, USA).

\section{Supplementary information}

Supplementary information accompanies this paper at https://doi.org/10 1186/s12870-019-2150-y.

Additional file 1: Table S1. Summary of sequencing reads after filtering. Detailed information, including the Illumina Q20 and Q30 quality scores of the transcripts, are shown in Table S1.

Additional file 2: Table S2. Primers used for $q R T-P C R$ analysis. The sequences of primers used for qRT-PCR are shown in Table S2.

Additional file 3: Figure S1. GO classification of DEGs. The $X$ axis represents the number of DEGs. The $Y$ axis represents the $\mathrm{GO}$ terms. 0-7 dai: comparison between 0 and 7 dai; 7-20 dai: comparison between 7 and 20 dai; 0-20 dai: comparison between 0 and 20 dai; dai: days after inoculation.

Additional file 4: Figure S2. Key regulatory points in the plant hormone signal transduction pathway. Pathway regulatory points containing genes that exhibit upregulated expression patterns in Cf-19 in the 0-7 dai and 0-20 dai comparisons (vertical comparisons, shown as four rectangles) or that exhibit upregulated expression patterns in CGN18423 plants compared with MM plants at the same interaction stages (lateral comparisons, shown as three rounds) were identified as important regulatory points. The yellow circle represents the vertical comparison result, and the blue circles represent the lateral comparison results.

Additional file 5: Figure S3. Key regulatory points in the plant pathogen interaction pathway. Pathway regulatory points containing genes that exhibit upregulated expression patterns in Cf-19 in the 0-7 dai and 0-20 dai comparisons (vertical comparisons, shown as four rectangles) or that exhibit upregulated expression patterns in CGN18423 plants compared with MM plants at the same interaction stages (lateral comparisons, shown as three rounds) were identified as important regulatory points. The yellow circle represents the vertical comparison result, and the blue circles represent the lateral comparison results.

\section{Abbreviations}

ABA: Abscisic Acid; Avr: Avirulence gene; BRI-1: BR-INSENSITIVE 1; BRs: Brassinosteroids; C. fulvum: Cladosporium fulvum; CaM: Calmodulin; 
cDNA-AFLP: cDNA-Amplified Fragment Length Polymorphism; CDPK: Calcium-Dependent Protein Kinase; CML: Calmodulin-Like; dai: days after inoculation; DEGs: Differentially Expressed Genes; ET: Ethylene; ETI: Effector-Triggered Immunity; Gas: Gibberellins; GO: Gene Ontology; Hcr2: Homologues of Cladosporium resistance gene Cf-2; Hcrg: Homologues of Cladosporium resistance gene Cf-9; HR: Hypersensitive Response; JA: Jasmonic Acid; JAZ: Jasmonate ZIM-domain; KEGG: Kyoto Encyclopedia of Genes and Genomes; LRR: Leucine-Rich Repeat; PAMP: Pathogen-Associated Molecular Pattern; PRRs: Pattern Recognition Receptors; PTI: PAMP-Triggered Immunity; qRT-PCR: Quantitative real-time PCR; R gene: Resistance gene; RLPs: Receptor-Like Proteins; SA: Salicylic Acid; TDFs: Transcript-Derived Fragments; TF: Transcription Factor; MAPK: Mitogen-Activated Protein Kinase; HSP90: Heat Shock Protein 90; QC: Quality Control; RSEM: RNA-Seq by Expectation Maximization; FPKM: Fragments Per Kilobase Per Million Mapped Reads; HISAT: Hierarchical Indexing for Spliced Alignment of Transcripts; LCMS: Liquid Chromatography-Mass Spectrometry; NCBI: National Center for Biotechnology Information; SGN: SOL Genomics Network

\section{Acknowledgements}

We would like to thank Mr. Guangliang Guo for his helpful assistance during the manuscript submission.

\section{Authors' contributions}

$T T Z$ and XXY designed the work and wrote the manuscript; DYZ, ZYW, YFB, $H H Y$ and $Y X$ performed the experiments; JBJ analysed the data; and HZ, QSC and JFL revised the work before submission; WHL and ZTZ performed final revision. All authors read, reviewed and approved the submitted version.

\section{Funding}

The design of this work was supported by the China Agriculture Research System (CARS-23-A-16) and the National Key R\&D Program of China (2017YFD0101900); Analysis and interpretation of data were funded by the Chinese Postdoctoral Science Foundation (2018 M630333), the Heilongjiang University Innovative Talents Training Plan (UNPYSCT-2018169), and the Science Foundation of Heilongjiang Province (C2017024); Writing the manuscript was funded by and the Outstanding Youth Science Fund of Northeast Agricultural University (17QC07).

\section{Availability of data and materials}

The clean data of all samples have been submitted to GenBank of the National Center for Biotechnology Information (NCBI, https://www.ncbi.nlm.nih.gov), and the Sequence Read Archive (SRA) accession number is SRP157120.

\section{Ethics approval and consent to participate}

Not applicable.

\section{Consent for publication}

Not applicable.

\section{Competing interests}

The authors declare that they have no competing interests.

\section{Author details}

${ }^{1}$ College of Horticulture and Landscape Architecture, Northeast Agricultural University, Harbin 150030, China. ${ }^{2}$ College of Agronomy, Northeast Agricultural University, Harbin, China. ${ }^{3}$ Key Laboratory of Biology and Genetic Improvement of Horticultural Crops (Northeast Region), Ministry of Agriculture and Rural Affairs, Harbin, China.

Received: 3 March 2019 Accepted: 19 November 2019 Published online: 19 December 2019

\section{References}

1. Thomma BP, Van Esse HP, Crous PW, De Wit PJ. Cladosporium fulvum, (syn. Passalora fulva), a highly specialized plant pathogen as a model for functional studies on plant pathogenic mycosphaerellaceae. Mol Plant Pathol. 2010:6:379-93.

2. Joosten M, De WP. The tomato-Cladosporium fulvum interaction: a versatile experimental system to study plant-pathogen interactions. Annu Rev Phytopathol. 1999;37:335-67.
3. Rivas S, Thomas CM. Molecular interactions between tomato and the leaf mold pathogen Cladosporium fulvum. Annu Rev Phytopathol. 2005:43:395436.

4. Jones JD, Dangl JL. The plant immune system. Nature. 2006;444:323-9.

5. De Wit PJGM, Mehrabi R, Van Den Burg HA, Stergiopoulos I. Fungal effector proteins: past, present and future. Mol Plant Pathol. 2009:10:735-47.

6. Rajamuthiah R, Mylonakis E. Effector triggered immunity: activation of innate immunity in metazoans by bacterial effectors. Virulence. 2014;5:697-702.

7. Hammondkosack KE, Jones JDG. Incomplete dominance of tomato Cf genes for resistance to Cladosporium fulvum. Mol Plant Microbe In. 1994;7:58-70.

8. Balint-Kurti PJ, Dixon MS, Jones DA, Norcott KA, Jones JDG. RFLP linkage analysis of the Cf-4 and Cf-9 genes for resistance to Cladosporium fulvum in tomato. Theor Appl Genet. 1994:88:691-700.

9. Kreuger J, Thomas CM, Golstein C, Dixon MS, Smoker M. A tomato cysteine protease required for $(f$-2-dependent disease resistance and suppression of autonecrosis. Science. 2002;296:744-7.

10. Koomangersmann M, Honee G, Bonnema G, De Wit P. A high-affinity binding site for the Avr9 peptide elicitor of Cladosporium fulvum is present on plasma membranes of tomato and other solanaceous plants. Plant Cell. 1996:8:929-38

11. Hong W, Xu YP, Zheng Z, Cao JS, Cai XZ. Comparative transcript profiling by CDNA-AFLP reveals similar patterns of Avr4/Cf-4- and Avr9/Cf-9-dependent defence gene expression. Mol Plant Pathol. 2010;8:515-27.

12. Cai $X$, Takken FLW, Joosten MH, Wit PJ. Specific recognition of Avr4 and Avr9 results in distinct patterns of hypersensitive cell death in tomato, but similar patterns of defence-related gene expression. Mol Plant Pathol. 2001;2:77-86.

13. Xue $\mathrm{D}$, Chen $\mathrm{X}$, Zhang $\mathrm{H}$, Chai $\mathrm{X}$, Jiang J. Transcriptome analysis of the $\mathrm{Cf}$ 12-mediated resistance response to Cladosporium fulvum in tomato. Front Plant Sci. 2017;7:2012.

14. Liu G, Liu J, Zhang C, You X, Zhao T. Physiological and RNA-seq analyses provide insights into the response mechanism of the $\mathrm{Cf}$-10-mediated resistance to Cladosporium fulvum infection in tomato. Plant Mol Biol. 2018:19:15.

15. JAL VK, GFJM VDA, De Wit PJ. Cloning and characterization of CDNA of avirulence gene Avr9 of the fungal pathogen Cladosporium fulvum, causal agent of tomato leaf mold. Mol Plant Microbe In. 1991;4:52-9.

16. Van Den Ackerveken GF, JAL VK, Joosten MH, Muisers JM, Verbakel HM, De Wit PJ. Characterization of two putative pathogenicity genes of the fungal tomato pathogen Cladosporium fulvum. Mol Plant Microbe In. 1993:6:210-5.

17. Joosten MH, Cozijnsen TJ, De Wit PJ. Host resistance to a fungal tomato pathogen lost by a single base-pair change in an avirulence gene. Nature. 1994;367:384-6.

18. Dixon MS, Jones DA, Hatzixanthis K, Ganal MW, Tanksley SD, Jones JD. Highresolution mapping of the physical location of the tomato Cf-2 gene. Mol Plant Microbe In. 1995;8:200-6.

19. Dixon MS, Jones DA, Keddie JS, Thomas CM, Harrison K, Jones JDG. The tomato $C f-2$ disease resistance locus comp rises two functional genes encoding leucine-rich repeat proteins. Cell. 1996:84:451-9.

20. Dixon MS, Hatzixanthis $K$, Jones DA, Harrison $K$, Jones JDG. The tomato Cf-5 disease resistance gene and six homologs show pronounced allelic variation in leucine-rich repeat copy number. Plant Cell. 1998;11:1915-25.

21. Thomas CM, Jones DA, Parniske M, Harrison K, Balint-Kurti PJ, Hatzixanthis K, Jones JDG. Characterization of the tomato Cf-4 gene for resistance to Cladosporium fulvum identifies sequences that determine recognitional specificity in Cf-4 and Cf-9. Plant Cell. 1997;9:2209-24.

22. Laugé $R$, Joosten $M H$, Haanstra JPW, Goodwin PH, Lindhout $P$, De Wit PJ. Successful search for a resistance gene in tomato targeted against a virulence factor of a fungal pathogen. Proc Natl Acad Sci U S A. 1998:95:9014-8.

23. Li S, Zhao TT, Li HJ, Xu XY, Li JF. First report of races 2.5 and 2.4 .5 of Cladosporium fulvum (syn. Passalora fulva), causal fungus of tomato leaf mold disease in China. J Gen Plant Pathol. 2015;81(2):162-5.

24. Bakker EG, Toomajian C, Kreitman M, Bergelson J. A genome wide survey of $R$ gene polymorphisms in A rabidopsis. Plant Cell. 2006;18:1803-18.

25. Laugé R, Joosten MH, Van Den Ackerveken GF, Van Den Broek HW, De Wit PJ. The in planta-produced extra cellular p roteins Ecp1 and Ecp2 of Cladosporium fulvum are virulence factors. Mol Plant Microbe In. 1997;10:725-34.

26. Laugé $\mathrm{R}$, Goodwin $\mathrm{PH}$, De Wit PJ, Joosten MH. Specific HR-associated recognition of secreted $p$ roteins from Cladosporium fulvum occurs in both host and non-host plants. Plant J. 2000:23(6):735-45.

27. Zhao T, Jiang J, Liu G, He S, Zhang H. Mapping and candidate gene screening of tomato Cladosporium fulvum-resistant gene Cf-19, based on high-throughput sequencing technology. BMC Plant Biol. 2016;16:51. 
28. Zhao T, Liu G, Li S, Li J, Jiang J. Differentially expressed gene transcripts related to the Cf-19-mediated resistance response to Cladosporium fulvum, infection in tomato. Physiol Mol Plant P. 2015;89:8-15.

29. Yang $H$, Zhao $T$, Jiang J, Chen $X$, Zhang $H$. Transcriptome analysis of the Sm-mediated hypersensitive response to Stemphylium lycopersici in tomato. Front Plant Sci. 2017:8:1257.

30. Xing M, Lv H, Ma J, Xu D, Li H. Transcriptome profiling of resistance to Fusarium oxysporum f. sp. conglutinans in cabbage (Brassica oleracea) roots. PLoS One. 2016;11:e0148048.

31. Chini A, Fonseca S, Fernández G, Adie B, Chico JM. The JAZ family of repressors is the missing link in jasmonate signalling. Nature. 2007;448:666-71.

32. Adie BAT, Perez-Perez J, Perez-Perez MM, Godoy M, Sanchez-Serrano JJ. ABA is an essential signal for plant resistance to pathogens affecting ja biosynthesis and the activation of defenses in Arabidopsis. Plant Cell Online. 2007;19:1665-81.

33. Robert-Seilaniantz A, Navarro L, Bari R, Jones JD. Pathological hormone imbalances. Curr Opin Plant Biol. 2007:10:372-9.

34. Cheval C, Aldon D, Galaud JP, Ranty B. Calcium/Calmodulin-mediated regulation of plant immunity. BBA-Mol Cell Res. 1833;2013:1766-71.

35. Downie AJ. Calcium signals in plant immunity: a spiky issue. New Phytol. 2015;204:733-5.

36. Morieri G, Martinez EA, Jarynowski A, Driguez H, Morris R. Host-specific nodfactors associated with $\backslash r$, Medicago truncatula $\backslash$, nodule infection differentially induce calcium influx and calcium spiking in root hairs. New Phytol. 2013;200:656-62.

37. Abramovitch RB, Anderson JC, Martin GB. Bacterial elicitation and evasion of plant innate immunity. Nat Rev Mol Cell Biol. 2006;7:601-11.

38. Heese A, Hann DR, Gimenez-Ibanez S, Jones AME, Rathjen JP. The receptorlike kinase SERK3/BAK1 is a central regulator of innate immunity in plants. $P$ Nati Acad Sci. 2007;104:12217-22

39. Gimenez-Ibanez S, Hann DR, Ntoukakis V, Petutschnig E, Lipka V. AvrPtoB targets the LysM receptor kinase CERK1 to promote bacterial virulence on plants. Curr Biol. 2009;19:423-9.

40. Wan J, Zhang X, Neece D, Ramonell KM, Clough SA. LysM receptor-like kinase plays a critical role in chitin signaling and fungal resistance in Arabidopsis. Plant Cell. 2008;20:471-81.

41. Wang X, Kota U, He K, Blackburn K, Li J. Sequential transphosphorylation of the BRI1/BAK1 receptor kinase complex impacts early events in brassinosteroid signaling. Dev Cell. 2008;15:0-235.

42. Menke FLH, Kang HG, Chen Z, Park JM, Kumar D. Tobacco transcription factor WRKY1 is phosphorylated by the MAP kinase SIPK and mediates HRlike cell death in tobacco. Mol Plant Microbe In. 2005;18:1027-34.

43. Andreasson $E$, Jenkins $T$, Brodersen $P$, Thorgrimsen $S$, Petersen NHT. The MAP kinase substrate MKS1 is a regulator of plant defense responses. EMBO J. 2005;24:2579-89.

44. Hsu FC, Chou MY, Chou SJ, Li YR, Peng HP, Shih MC. Submergence confers immunity mediated by the WRKY22 transcription factor in Arabidopsis. Plant Cell. 2013;25:2699-713.

45. Lippok B, Birkenbihl RP, Rivory G, Brümmer J, Somssic IE. Expression of AtWRKY33 encoding a pathogen-or PAMP-responsive WRKY transcription factor is regulated by a composite DNA motif containing W box elements. Mol Plant Microbe In. 2007;20:420-9.

46. Cui $H$, Wang $Y$, Xue L, Chu J, Yan C. Pseudomonas syringae effector protein AvrB perturbs Arabidopsis hormone signaling by activating MAP kinase 4. Cell Host Microbe. 2010;7:164-75.

47. Bigeard J, Colcombet J, Heribert H. Signaling mechanisms in patterntriggered immunity (PTI). Mol Plant. 2015;8:521-39.

48. Zhang W, Song W, Zhang ZQ, Wang HD, Yang MM, Guo RJ, Li ML. Transcriptome analysis of Dastarcus helophoroides (Coleoptera: Bothrideridae) using Illumina HiSeq sequencing. PLoS One. 2014;9: e100673.

49. Trapnell C, Williams BA, Pertea G, Mortazavi A, Pachter L. Transcript assembly and quantification by RNA-seq reveals unannotated transcripts and isoform switching during cell differentiation. Nat Biotechnol. 2010; 28:511-5.

50. Dewey CN, Bo L. RSEM: accurate transcript quantification from RNA-seq data with or without a reference genome. BMC Bioinformatics. 2011;12:323.

51. Kim D, Langmead B, Salzberg SL. HISAT: a fast spliced aligner with low memory requirements. Nat Methods. 2015;12:357-60.

52. Anders $\mathrm{S}$, Huber W. Differential expression analysis for sequence count data. Genome Biol. 2010;11:106.
53. Tarazona S, Furió-Tarí $P$, Turrà D. Data quality aware analysis of differential expression in RNA-seq with NOISeq r/Bioc package. Nucleic Acids Res. 2015; 43(21):e140 gkv711.

54. Benjamini Y, Hochberg Y. Controlling the false discovery rate: a practical and powerful approach to multiple testing. J R Stat Soc B. 1995;57:289-300.

55. Pertea M, Pertea GM, Antonescu CM, Chang TC, Mendell JT. Stringtie enables improved reconstruction of a transcriptome from RNA-seq reads. Nat Biotechnol. 2015;33:290-5.

56. Chen ZZ, Xue CH, Zhu S, Zhou FF, Ling XB, Liu GP, Chen LB. Go pipe: streamlined gene ontology annotation for batch anonymous sequences with statistics. Prog Biochem Biophys. 2005;32:187-91.

57. Kanehisa M, Goto S, Furumichi M, Tanabe M, Hirakawa M. KEGG for representation and analysis of molecular networks involving diseases and drugs. Nucleic Acids Res. 2010;38:355-60.

58. Livak KJ, Schmittgen TD. Analysis of relative gene expression data using real-time quantitative PCR and the $2^{-\Delta \Delta C T}$ method. Methods. 2001;25:402-8.

59. Rotenberg D, Thompson TS, German TL, Willis DK. Methods for effective real-time RT-PCR analysis of virus-induced gene silencing. J Virol Methods. 2006:138:49-59.

60. Llugany M, Martin SR, Barceló J, Poschenrieder C. Endogenous jasmonic and salicylic acids levels in the cd-hyperaccumulator Noccaea (Thlaspi) praecox exposed to fungal infection and/or mechanical stress. Plant Cell Rep. 2013; $32: 1243-9$.

\section{Publisher's Note}

Springer Nature remains neutral with regard to jurisdictional claims in published maps and institutional affiliations.
Ready to submit your research? Choose BMC and benefit from:

- fast, convenient online submission

- thorough peer review by experienced researchers in your field

- rapid publication on acceptance

- support for research data, including large and complex data types

- gold Open Access which fosters wider collaboration and increased citations

- maximum visibility for your research: over 100M website views per year

At BMC, research is always in progress.

Learn more biomedcentral.com/submissions 